\title{
Selected dynamic anthropometrics and body characteristics for posture corrector fit
}

\author{
Inga Dāboliṇa1*, Jelizaveta Fomina², Eva Lapkovska², Liene Siliṇa² \\ ${ }^{1}$ Head of Research Laboratory of Ergonomics Electrical Technologies, Institute of Industrial Electronics and \\ Electrical Engineering, Faculty of Electrical and Environmental Engineering, Riga Technical University, Riga, Latvia \\ 2 Institute of Design Technologies, Faculty of Material Science and Applied Chemistry, Riga Technical University, \\ Riga, Latvia \\ *Corresponding author E-mail address: inga.dabolina@rtu.lv
}

\section{INFO}

CDAPT, ISSN 2701-939X

Peer reviewed article

2020, Vol. 1, No. 2, pp. 96-103

DOI 10.25367/cdatp.2020.1.p96-103

Received: 12 July 2020

Accepted: 11 November 2020

Available online: 22 November 2020

\section{Keywords}

ergonomics,

anthropometrics,

posture,

posture corrector,

compression garment,

human body $3 \mathrm{D}$ scanning

\begin{abstract}
Correct body posture is a balanced musculoskeletal body position; however, today many people face severe posture defects, and their body posture may be far from normal, causing progressive musculoskeletal deformities and pain, as well as affecting the functionality and appearance of the body. Human daily habits, lack of physical activities and overall a sedentary lifestyle cause such phenomena. The research focuses on studies of human body measurements and body characteristics while wearing posturecorrective equipment for the assessment of the effectiveness of different posture correctors $(P o C)$. Four test-persons were selected and scanned using $3 D$ anthropometrical scanner in relaxed stand position wearing five different posture correctors for a prolonged period of time in order to determine the effectiveness and functionality of each PoC. Four distance (linear) human body measurements were gained to quantify postural changes scapula position depth, upper torso position, shoulder projection height and hip-waist depth. General feedbacks on each subject impression of wearing PoCs were also received. Correction of posture could serve as a preventive or treatment for spine-related problems; though, commercially available correctors could negatively affect wearer's health due to non-conformity of the design and/or used material. Therefore, PoC selection and wearing procedures should be solved with more personalized and customized approaches.
\end{abstract}

(C) 2020 The authors. Published by CDAPT.

This is an open access article under the CC BY-NC-ND license https://creativecommons.org/licenses/ peer-review under responsibility of the scientific committee of the CDAPT. 


\section{Introduction}

Almost every person faces posture defects. The main reason that leads to the wrong posture and resulting health problems is back muscle weakness caused by lack of body movements or physical activities (unless postural defects are congenital or caused by disease). The human body movement system is strengthened insufficiently due to the lack of physical activities. One of the symptoms is back pain, though discovering the cause of it is not always easy. Normalization of posture could serve as a preventive measure or treatment for back pain or sprains [1-3]. The main functions of posture correctors (PoCs) are to correct body posture, as well as to relieve back and neck pain [1]. Mostly such PoCs are intended to help a person maintain a correct body posture and, if necessary, for spinal support. The most obvious method for determination of posture garment effects on wearer's posture is the assessment of postural changes [4-7].

There are various types of belts, bandages, back supports and posture correctors available on the market that could help with correcting posture defects. However, the use of such commercially available products daily could be cumbersome due to unsuitable choice of textiles and design deficiencies; moreover, they could cause skin irritation and limited mobility [6]. To evaluate the effects of different PoC products, four test-persons were selected to test and analyze five posture correctors.

\section{Posture disorders and means of their correction}

Posture is defined as the relative position of body parts to a physical position, such as standing, lying, and sitting. Correct posture - a straight spine in relation to the medial plane that supports the natural curve of the spine, maintaining the balance of muscles and skeleton [4]. This balanced musculoskeletal position protects the supporting structures of the body and prevents damage or progressive deformation in all positions, including standing, lying, and sitting $[2,4]$.

The importance of the right body posture and particularly emphasizing the right posture while sitting should be promoted. In most cases, the occurrence of poor posture is affected by human habits, such as back bending or leg crossing, sedentary work, use of smart devices, and generally sessile lifestyle without proper physical activities. If taking a poor posture becomes a habit at an early age, a person who maintains this posture becomes accustomed to it and even feels comfortable, thus causing tension in the spine, pelvis, muscles, tendons, joints, bones and discs, leading to fatigue and spine deformations [8]. Spinal injuries mostly occur in young and actively working people (as a result of high traumatic energies), relatively less often in older people with osteoporosis (as a result of minimal influencing energies).

\subsection{Posture disorders}

The spine of an adult has the shape of the letter "S": in the neck and lumbar region the spine is curved forward (lordosis), while in the chest and sacrum the spine forms two curves backwards (kyphosis). Due to its curved shape, the spine can absorb pushes and convulsions, and therefore it oscillates flexibly as a person walks, jumps and runs. From a physical medicine point of view, a posture is a balance of muscles and skeleton that protects the body's supporting structures from damage or progressive deformation regardless of its position (lying, sitting on a squat or leaning), in which these structures work or rest [8]. Posture asymmetries can be progressive and affect functional activities, as maintaining the wrong posture for a prolonged period of time causes significant strain on the spine [9-12]. Postural disorders can be caused by a variety of reasons:

spinal asymmetries (scoliosis, kyphosis, lordosis)

injuries/traumas (clavicle injury, axial skeleton injury, et al.)

diseases (Scheuermann's disease, osteoporosis, et at.). 


\subsection{Posture correctors}

Various methods are used to treat diseases of the spine and joints, including orthopedic products called orthoses. Orthoses are functional orthopedic aids to change the structural and functional properties of the musculoskeletal system, to immobilize, relieve or correct a diseased spine or limb, including various medical bandages, collars, corsets, belts, etc. The main task of orthoses is to ensure temporary and secure immobilization of certain muscles and skeletal system segments, as well as to compensate functionally deficient limbs and body parts. The period of wearing the orthoses is regulated by a doctor, and they can be produced individually or in series $[9,13]$. Orthoses (belts/corsets/vests) are used to reduce pain, protect against further damage, help weak muscles, and prevent or help to correct the deformity by maintaining torso alignment through passive force $[13,14]$.

Various means are used to correct posture and relieve back pain:

sitting support devices (cushions, Swiss ball chairs, et al.)

kinesiology tape

Posture braces/Orthosis (full spine brace/reclinator, upper back brace/cross-back elastic brace, electronic posture reminder, et al.).

The variety of patents includes various posture correction solutions from textiles or partial use of textiles - such as posture correction girdles [15], spinal orthoses [16,17], posture vests [18], therapeutic garment systems [19] and other posture support devices [20,21,22].

In this study five posture correctors - three upper back brace and two full spine brace - were analyzed, see Fig. 1:

PoC \# 1 cross-back elastic brace with wide straps

PoC \# 2 cross-back elastic brace with narrow straps

PoC \# 3 cross-back elastic brace with wide hemmed straps and narrowing of the straps in the area of the shoulders and armpits

PoC \# 4 reclinator with half waist belt

PoC \# 4 reclinator with full waist belt.

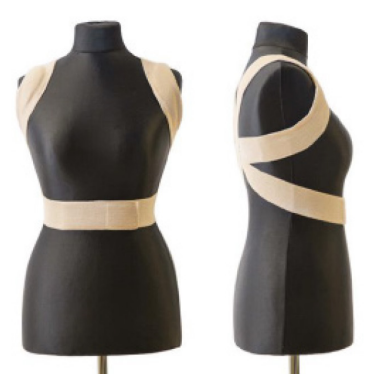

$\mathrm{a}-\mathrm{PoC} \# 1$
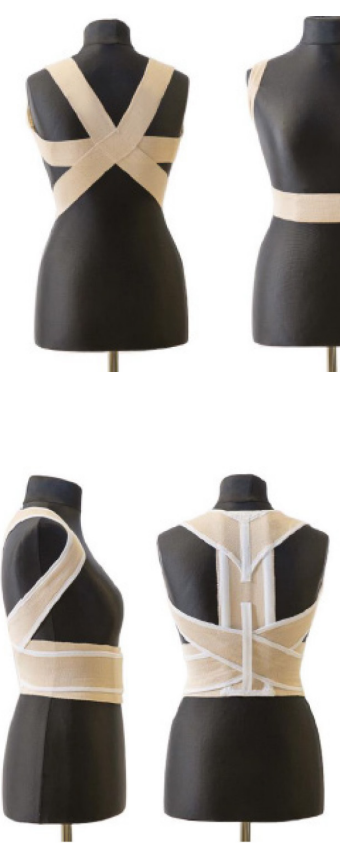

d - PoC\#4

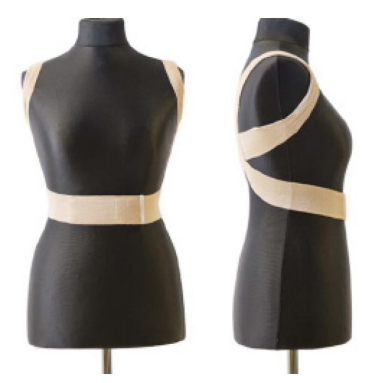

b - PoC\#2
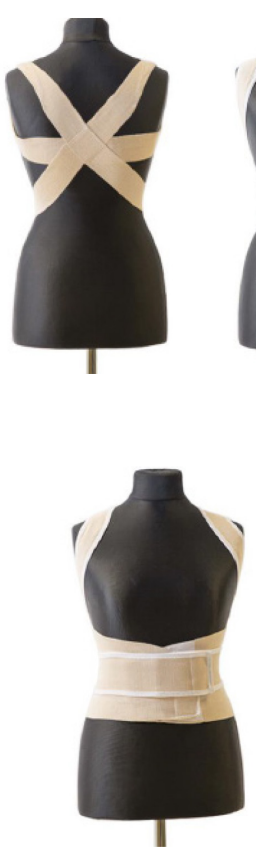
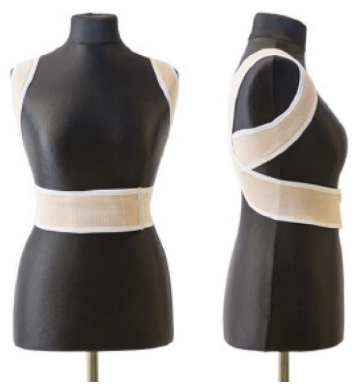

c- PoC\#3
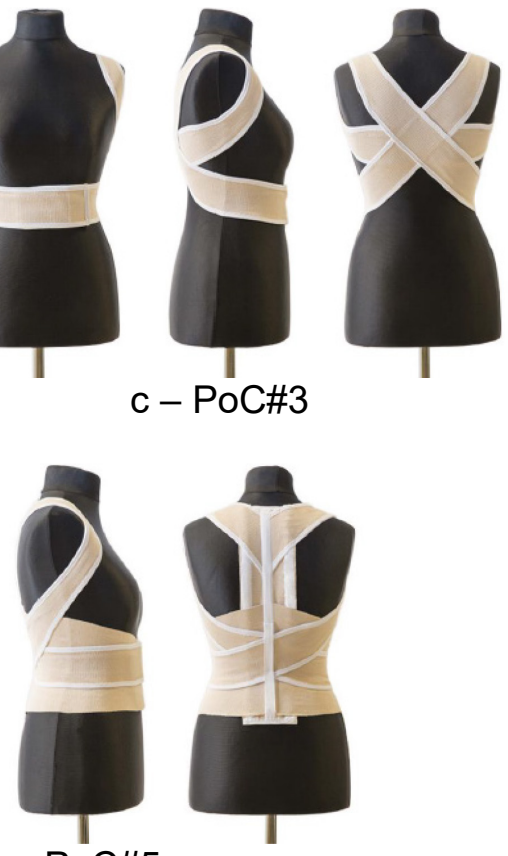

e - PoC\#5

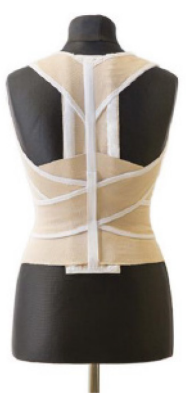

Fig. 1 (a-c) Upper back brace; $(d, e)$ full spine brace. 


\section{Testing of posture correctors \\ 3.1 Method}

Test subjects were scanned using 3D human body scanning device Vitus Smart XXL ${ }^{\circledR}$. The scanning was performed six times in a time frame of one work-day for each posture corrector: at the beginning of the day without posture corrector and with posture corrector, three times every two hours while wearing posture corrector, the last time - after wearing period of six hours - without posture corrector. Each scanning set was conducted as follows: one set consisted of three scans; after each scan the test subject was asked to step off the scanner platform and step again back on it. All participants were scanned while wearing their underwear and standing in a natural/relaxed posture.

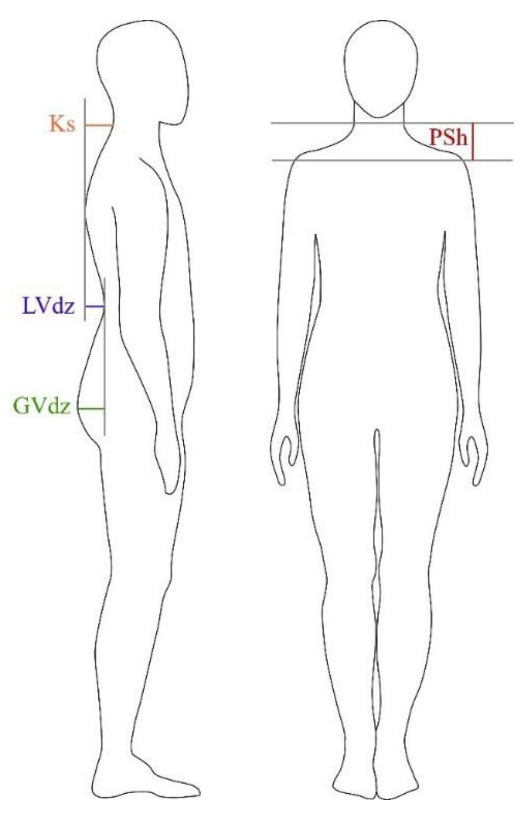

Fig. 2 Measurements determining the change of posture.

Four linear human body measurements were selected to determine postural changes - scapula position depth (LVdz), upper torso position (Ks), shoulder projection height (Psh) and hip-waist depth (GVdz), see Fig. 2. The obtained measurements were summarized, and the median value was selected (from all three scans of each test set). The use of an arithmetic mean value was not appropriate due to the uniformity of the human body. Selecting values that did not correspond in any of the cases was not in accordance with the methodology; therefore a scan with medial measurements from all three measurements was accepted as the calculation model.

The analysis of the data took into account both - the effect of each posture corrector on the test subject and the effect on individual measurements.

\subsection{Test-persons}

To determine the effects of each PoC on wearer's posture, four test subjects were selected - women aged 25 to 35 years (each was assigned a letter designation: $A$ to D). All test subjects have a slender body structure (see Table 1) and moderately developed musculature. The test-person A has no posture or spine defects, is physically well developed, but not trained. As for other test subjects, this person has mild back problems - the prominence of the scapula, scoliotic and/or kyphotic changes.

Test-person A was a reference test-person without back problems and with the best trained (but not athlete's) body; respectively, posture certainty and repeatability for this person was the highest, while test-person $\mathrm{C}$ had the most uncertain physical fitness and could be the most easily influenced, and posture correctors are expected to give the best result to test-person C. 
Table 1. Characteristics of test-persons.

\begin{tabular}{lllll}
\hline Person: & A & B & C & D \\
\hline Body height $(\mathrm{cm})$ & 173 & 159 & 167 & 164 \\
Horizontal bust girth $(\mathrm{cm})$ & 97 & 88 & 93 & 90 \\
Hip girth $(\mathrm{cm})$ & 95 & 93 & 93 & 93 \\
Weight $(\mathrm{kg})$ & 64 & 51 & 58 & 51 \\
\hline
\end{tabular}

\subsection{Analysis of data}

After testing the posture correctors, graphs were drawn up for each test subject, including all stages (scanning sets) of the experiment, showing the changes of the four previously selected human body measurements (LVdz, Ks, Psh, GVdz) (see Fig. 3).

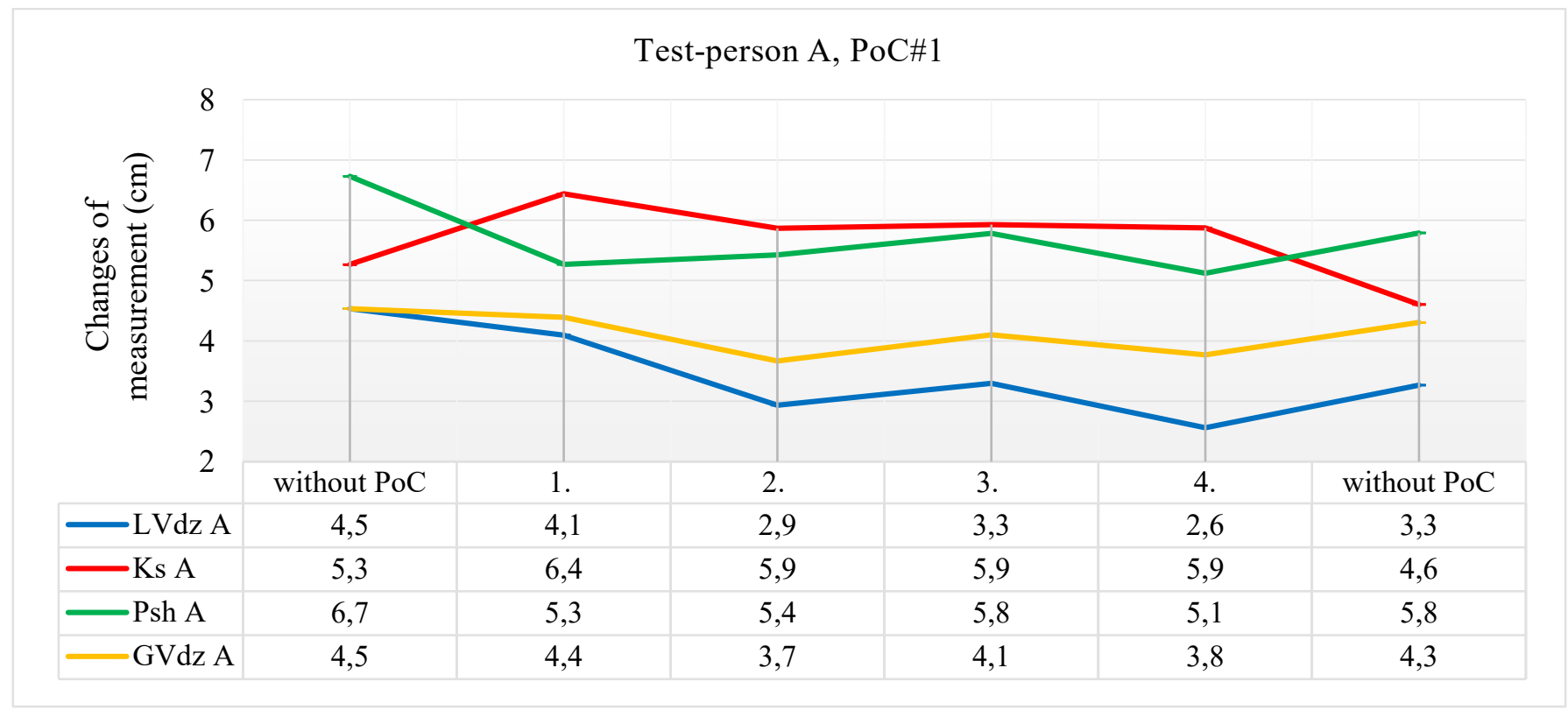

Fig. 3 Effect of PoC\#1 to the test-person A.

According to the change in the measurement visible from the graph, the effect of PoC was evaluated in comparison to the first scan without PoC. If the value is greater than or equal to the original (a positive value indicates a bent posture, a negative value indicates that no change was observed), then the stage was marked with 0 , if less, then with 1 . The obtained binary scores were summed to describe the extent to which the PoC affects the particular measurement. The last column "without PoC" determines how the test-subject's posture as a whole was affected by evaluating it without posture corrector at the end of the experiment - positively or negatively according to the same comparison method (see Table 2).

Table 2. Analysis of effect of PoC\#1 to the test-person A

\begin{tabular}{|c|c|c|c|c|c|c|}
\hline $\begin{array}{ll}\text { Measurement } & \text { Test \# } \\
\end{array}$ & 1. & 2. & 3. & 4. & $\Sigma$ & $\begin{array}{l}\text { without } \\
\text { PoC }\end{array}$ \\
\hline LVdz & 1 & 1 & 1 & 1 & 4 & 1 \\
\hline Ks & 0 & 0 & 0 & 0 & 0 & 1 \\
\hline Psh & 1 & 1 & 1 & 1 & 4 & 1 \\
\hline GVdz & 1 & 1 & 1 & 1 & 4 & 1 \\
\hline
\end{tabular}

Such comparison method was used for all measurements and the overall impact of PoC. After a binary comparison which is to assess all properties in pairs - if one is evaluated as better than other, it is 
marked as 1 , if properties (PoCs) are with equal impact, both are marked as 0.5 (see Table 3 ), it was concluded that regardless of whether or not there is a narrowing of the straps in the area of shoulders and armpits, PoC \# 1 and PoC \# 2 affected the test subjects in the same way and that the result for each test-person was more dependent on their physiological features.

To support the data, a posture test was performed on test subjects $A$ and $C$ without PoC. The experimental procedure was the same as using PoC. These tests showed only insignificant changes in stature (also posture), which confirms the effect of PoC on test subjects.

Table 3. Binary comparison of the effect of PoC on the test-person.

\begin{tabular}{|c|c|c|c|c|c|c|c|}
\hline \multicolumn{2}{|c|}{ Effect on the test-person } & \multirow{2}{*}{ PoC\#1 } & \multirow{2}{*}{$\begin{array}{l}\text { PoC\#2 } \\
1\end{array}$} & \multirow{2}{*}{$\begin{array}{l}\text { PoC\#3 } \\
1\end{array}$} & \multirow{2}{*}{$\begin{array}{l}\text { PoC\#4 } \\
1\end{array}$} & \multirow{2}{*}{$\begin{array}{l}\text { PoC\#5 } \\
1\end{array}$} & \multirow{2}{*}{$\begin{array}{l}\Sigma \\
4\end{array}$} \\
\hline PoC\#1 & person $A$ & & & & & & \\
\hline PoC\#2 & person A & 0 & & 0 & 0 & 0 & 0 \\
\hline PoC\#3 & person $A$ & 0 & 1 & & 0.5 & 1 & 2.5 \\
\hline PoC\#4 & person $A$ & 0 & 1 & 0.5 & & 1 & 2.5 \\
\hline PoC\#5 & person $A$ & 0 & 1 & 0 & 0 & & 1 \\
\hline PoC\#1 & person B & 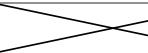 & 0 & 0 & 0 & 0.5 & 0.5 \\
\hline PoC\#2 & person B & 1 & & 1 & 1 & 1 & 4 \\
\hline PoC\#3 & person B & 1 & 0 & & 0.5 & 1 & 2.5 \\
\hline PoC\#4 & person $B$ & 1 & 0 & 0.5 & & 1 & 2.5 \\
\hline PoC\#5 & person B & 0.5 & 0 & 0 & 0 & & 0.5 \\
\hline PoC\#1 & person C & 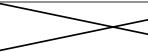 & 0.5 & 0 & 0.5 & 0 & 1 \\
\hline PoC\#2 & person $\mathrm{C}$ & 0.5 & & 0 & 0.5 & 0 & 1 \\
\hline PoC\#3 & person C & 1 & 1 & & 1 & 0.5 & 3.5 \\
\hline PoC\#4 & person $C$ & 0.5 & 0.5 & 0 & & 0 & 1 \\
\hline PoC\#5 & person $\mathrm{C}$ & 1 & 1 & 0.5 & 1 & + & 3.5 \\
\hline PoC\#1 & person $D$ & 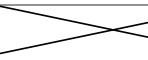 & 1 & 1 & 1 & 0.5 & 3.5 \\
\hline PoC\#2 & person $D$ & 0 & & 1 & 0.5 & 0 & 1.5 \\
\hline PoC\#3 & person $D$ & 0 & 0 & & 0 & 0 & 0 \\
\hline PoC\#4 & person $D$ & 0 & 0.5 & 1 & & 0 & 1.5 \\
\hline PoC\#5 & person $D$ & 0.5 & 1 & 1 & 1 & & 3.5 \\
\hline
\end{tabular}

According to the binary comparison and its summary results, it could be concluded that there were no favorites among posture correctors - each PoC reacts to each test-person differently.

Similar conclusions could be drawn from listening to test subjects, for example, test subject $A$ had a good impression of the effects of PoC \# 1 on posture and well-being, while test subject $C$ immediately felt uncomfortable when putting on PoC \# 1. The same could be said for all PoCs.

\section{Conclusions}

Based on the medical literature and research on posture improvement, it has been concluded that an ideal or correct posture requires a balanced body position and proportion. Today, on the other hand, people's social and private lives are dominated by bad habits, such as bending over while studying and working, using computers and smart devices too often, carrying heavy bags, using desks and stools that are not appropriate in height for a person's body, lack of health education and physical activity. All these factors affect muscle shape, deform skeleton and cause malformations that prevent proper posture. A good, correct posture helps a person to participate in active and healthy life, as well as to achieve a good body appearance.

Compression of soft tissues while wearing a posture corrector negatively affects certain aspects of the normal psychological balance of the human body and can also become a health risk factor. When choosing $\mathrm{PoC}$, the patient must pay attention to the design aspects of $\mathrm{PoC}$ that could determine wearing 
comfort - narrowing of the straps in the area of shoulders and armpits. To avoid skin irritation, PoC is recommended to be worn on tight-fitting garments.

Measurements confirm that the proportional relationships of human lordosis and kyphosis do not change correlatively; therefore, spinal change analysis is a task with a lot of room for data interpretation. Regardless of the design (pattern) and type of PoC, the back muscles relax after just a few hours, which rapidly reduces the product's effectiveness and functional properties, meaning that not only the PoC itself must be personalized, but also the wearing time, regularity and intensity must be coordinated for each individual.

Future studies should include a method to correlate test-persons' views with the effects of the tested PoC on a person's posture. The trend of the currently analyzed statements suggests that if the test subject has psychological and physiological objections, this results in a worse effect of PoC on the test subject's posture corrections. To refine the experimental data, the measurements should be repeated under the same conditions for several days to exclude the effects of a particular mood. Such measurements are very time consuming and place limitations on test subjects to perform their casual routine duties.

\section{Acknowledgements}

This work has been supported by the European Regional Development Fund within the Activity 8.2.2 "To Strengthen Academic Staff of Higher Education Institutions in the Areas of Strategic Specialization".

\section{References}

[1] Saemee, L. Posture Modification Effects Using Soft Materials Structures. Dissertation, University of Minnesota, Minneapolis, USA, 2016.

[2] Kim, D.; Cho, M.; Park, Y.; Yang, Y. Effect of an exercise program for posture correction on musculoskeletal pain. J. Phys. Ther. Sci., 2015, 27 (6), 1791-1794. DOI: 10.1589/jpts.27.1791.

[3] Watkins, S. M.; Dunne, L. E. Functional clothing design. From sportswear to spacesuits. Bloomsbury, 2015.

[4] Lyu, S.; LaBat, K. "Shapewear" for women as a postural improvement garment. International Textile and Apparel Association Annual Conference Proceedings, 2014, 71 (1), 1-2.

[4] Liu, P. Y.; Yip, J.; Yick, K. L.; Yuen, C. W. M.; Tse, C. Y.; Ng, S. P.; Law, D. Effects of a tailor-made girdle on posture of adolescents with early scoliosis, Textile Research Journal, 2015, 85 (12), 1234-1246. DOI: https://doi.org/10.1177/0040517514561928.

[5] Liu, S. The effects of an innovative posture garment technology on the scapular and lumbar muscle activity and scapular alignment. International Journal of Physical Medicine \& Rehabilitation, 2018, 6, p.14.

[6] McRoberts, L. B.; Black, C. M.; Cloud, R. M. Evaluation of a prototype soft-structured thoracic posture support garment. Clothing and Textiles Research Journal, 2016, 34 (2), 143-158. DOI: 10.1177/0887302X15622571.

[7] McRoberts, L. B., et al. Evaluation of the New York posture rating chart for assessing changes in postural alignment in a garment study. Clothing and Textiles Research Journal, 2013, 31, 81-96. DOI: $10.1177 / 0887302 X 13480558$.

[8] Kendall, F. P.; McCreary, E. K.; Provance, P. G.; Rodgers, M. M.; Romani, W. A. Muscles: Testing and function with posture and pain, $5^{\text {th }}$ ed.; Lippincott Williams \& Wilkins: Baltimore, MD, USA, 2005, 512.

[9] Kouwenhoven, J. W; Castelein, R. M. The pathogenesis of adolescent idiopathic scoliosis: review of the literature. Spine, 2008, 33 (26), 2898-2908. DOI: 10.1097/BRS.0b013e3181891751.

[10] Chow, D. H.; Kwok, M. L.; Cheng, J. C.; Lao, M. L. Holmes, A. D., Au-Yang, A., Wong, M. S. The effect of backpack weight on the standing posture and balance of schoolgirls with adolescent idiopathic scoliosis and normal controls. Gait Posture, 2006, 24 (2), 173-181. DOI: 10.1016/j.gaitpost.2005.08.007.

[11] Mahaudens, P.; Thonnard, J. L.; Detrembleur, C. Influence of structural pelvic disorders during standing and walking in adolescents with idiopathic scoliosis. The Spine Journal, 2005, 5(4), 427-433. DOI 10.1016/j.spinee.2004.11.014.

[12] Jaworski, P. Postural defects - correct posture, pathological posture, pathogenesis and consequences, Journal of education, health and sport, 2019, 9 (8), 452-460.

[13] ISO 13404:2007 "Prosthetics and orthotics - Categorization and description of external orthoses and orthotic components". 
[14] Bazzarelli, M.; Durdle, N.; Lou, E.; Raso, J. A low power portable electromagnetic posture monitoring system. Conference Proceedings (Cat. No.01TH8555) of Canadian Conference on Electrical and Computer Engineering 2001, Toronto, Ontario, Canada, 2001, Vol. 2, 1373-1377. DOI: 10.1109/CCECE.2001.933652.

[15] Yip, Y. W. J.; Yick, K. L.; Tse, C. Y.; Yuen, C. W. M.; Ng, S. P.; Liu, P. Y.; Law, K. M., inventors, Hong Kong Polytechnic University HKPU, assignee, Posture correction girdle and the method of correcting spinal deformity, United States Patent 9,398.972 B2, Jul. 26, 2016.

[16] Mills, A. J., inventor, SPINECORPORATION Ltd., assignee, Spinal orthosis, United States US 2010/0217166 A1, Aug. 26, 2010.

[17] Ingimundarson, A. T.; Bakken, N.; Omarsson, B.; Romo, H. D.; Nemeth, J., inventors, Ossur hf, assignee, Spinal orthosis, United States US 2017/0156911 A1, Jun. 8, 2017.

[18] Lai, W.; Mao, K., inventors, Mao, K.; Lai, W. C., assignees, Posture vest, United States Patent US 7,842,000 B2, Nov.30, 2010.

[19] Taylor, B. C., inventor, Thera Togs Inc., assignee, Elasticized garment and strapping system to aid in body mobility support and maintenance, United States Patent US 8,007,457 B2, Aug. 30, 2011.

[20] Pollack, J., inventor, Pollack, J., assignee, System, method, and device for posture support, United States Patent US 9,456,919 B2, Oct. 4, 2016.

[21] Brown T. W., inventor, ALIGNMED Inc., assignee, Posture improvement device and method of use, United States US 2011/0213283 A1, Sep. 1, 2011.

[22] Ingimundarson, A. T.; Robertson, B., inventors, Ossur hf, assignee, Orthopedic device for treatment of the back, United States Patent US 9,414,953 B2, Aug. 16, 2016. 